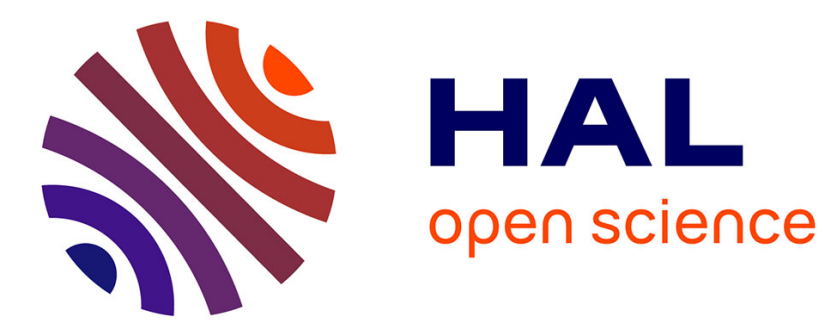

\title{
The Dynamics of Lobbying under Uncertainty: On Political Liberalization in Arab Countries
}

\author{
Raouf Boucekkine, Fabien Prieur, Klarizze Puzon
}

\section{To cite this version:}

Raouf Boucekkine, Fabien Prieur, Klarizze Puzon. The Dynamics of Lobbying under Uncertainty: On Political Liberalization in Arab Countries. 2013. halshs-00801961

\section{HAL Id: halshs-00801961 https://shs.hal.science/halshs-00801961}

Preprint submitted on 18 Mar 2013

HAL is a multi-disciplinary open access archive for the deposit and dissemination of scientific research documents, whether they are published or not. The documents may come from teaching and research institutions in France or abroad, or from public or private research centers.
L'archive ouverte pluridisciplinaire HAL, est destinée au dépôt et à la diffusion de documents scientifiques de niveau recherche, publiés ou non, émanant des établissements d'enseignement et de recherche français ou étrangers, des laboratoires publics ou privés. 


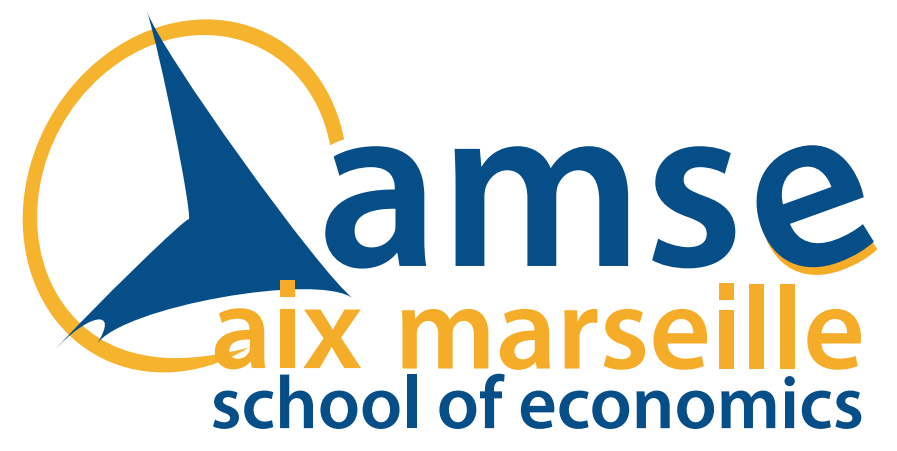

\section{Working Papers / Documents de travail}

The Dynamics of Lobbying under Uncertainty: On Political Liberalization in Arab Countries

Raouf Boucekkine

Fabien Prieur

Klarizze Puzon 


\title{
The dynamics of lobbying under uncertainty: on political liberalization in Arab countries*
}

\author{
Raouf Boucekkine ${ }^{\dagger}$ Fabien Prieur ${ }^{\ddagger}$, and Klarizze Puzon ${ }^{\S}$
}

March, 152013

\begin{abstract}
We consider a framework à la Wirl (1994) where political liberalization is the outcome of a lobbying differential game between a conservative elite and a reformist group, the former player pushing against political liberalization in opposition to the latter. In contrast to the benchmark model, we introduce uncertainty. We consider the typical case of an Arab oil exporter country where oil rents are fiercely controled by the conservative elite. We assume that the higher the oil rents, the more reluctant to political liberalization the elite is. Two states of nature are considered (high vs low resource rents). We then compute the Market-perfect equilibria of the corresponding piecewise deterministic differential game. It is shown that introducing uncertainty in this manner increases the set of strategies compared to Wirl's original setting. In particular, it is shown that the cost of lobbying might be siginificantly increased under uncertainty with respect to the benchmark. This ultimately highlights some specificities of the political liberalization at stake in Arab countries and the associated risks.
\end{abstract}

Keywords: Rent-seeking, lobbying, natural resources, Arab countries, piecewise deterministic differential games

JEL classification: D72, C61, C73

*Raouf Boucekkine acknowledges financial support from the French research ageny ANR, grant ANR-12-GLOB-0005-01. The usual disclaimer applies.

${ }^{\dagger}$ Corresponding author. Aix-Marseille University (Aix-Marseille School of Economics), CNRS \& EHESS, and IRES-CORE, Universite catholique de Louvain. Address: GREQAM, 2 rue de la charité, 13002 Marseille(France). E-mail: raouf.boucekkine@univ-amu.fr

‡LAMETA, Universite Montpellier I and INRA. E-mail: prieur@supagro.inra.fr

$\S$ LAMETA, Universite Montpellier I. E-mail: klarizze.puzon@lameta.univ-montp1.fr 


\section{Introduction}

Rent-seeking activities in countries with developed extraction sectors are abundantly documented. Examples range from timber industries in the Philippines and Malaysia (as detailed in Ross, 2001) to fossil energy-related sectors like in OPEC countries (see a recent paper by Gylfason, 2001). In general, the rents deriving from the exploitation of natural resources fall under the fierce control of conservative elites. These elites typically manipulate national legislation (pretty much in the sense given by Tullock (1967) to rent-seeking) to perpetuate themselves in power 1 Empirical evidence show that the so-called "resource curse" can be a consequence of the latter behaviour. Bad governance and weak institutions are the main reasons behind the failures of several resource-rich countries to launch a sustainable growth process (see Gylfason, 2001, and more recently, Mehlum et al., 2006). The "resource curse" is by no way the mere outcome of an automatic mechanism penalizing these otherwise blessed countries.

On the other hand, the impact of rent-seeking behaviour on economic efficiency is a quite old idea tracing back to Tullock (1967) as already mentioned above. Key aspects of the theory are the strategic and non-strategic behaviours of the players involved in rent-seeking and their impact (and interaction) with public policy. As players are roughly the representatives of interest groups in practice, the theory ends up modelling the determinants and outcomes of lobbying in different theoretical contexts (see Becker (1983), Linster (1994), and Kohli and Singh (1999) for more recent examples of the literature stream opened by Tullock). An influential contribution is the one by Becker (1993). He modeled lobbying in a two-player setting, each player with his own lobbying cost and productivity. It was assumed that the larger lobbying expenditures, the stronger the lobby and the more effective a player can be in orienting public (fiscal) policy. However, Becker's model does not entail any strategic behaviour of any sort: each player acts as if the lobbying effort exerted by the opponent is independent of his own choice $2^{2}$ Researchers after Becker have tried to get rid of this shortcoming. To our knowledge, Wirl (1994) is the first to use differential games

\footnotetext{
${ }^{1}$ The recent Arab Spring uprising shed light on another form of these long lasting rentseeking activities, not related to natural resources but to the control of financial and trade flows as it was the case in Tunisia under the presidency of Benali.

${ }^{2}$ The main point made by Becker is that increasing competition among interest groups should improve the efficiency of the tax system.
} 
in this stream of literature. Wirl uses a linear-quadratic model to investigate the impact of the game structure on the outcomes expressed in terms of players' strategies. Though the government is passive in this framework (in other words, public policy only changes in response to lobbying actions), the paper has two important contributions. First, the game structure matters (the open-loop equilibria are, indeed, carefully compared to the subgame-perfect equilibria derived as linear Markov strategies). Second, in the subgame-perfect equilibria, optimal lobbying expenditures are remarkably lower (than those observed in the openloop case). This provides a rationale for a conjecture made by Tullock. The cost of rent-seeking activities are rather small compared to the rents, therefore implying not too high social costs. The reason behind this striking result is inherent in the feedback nature of the Markovian strategies, which discourages too aggressive lobbying strategies (see Wirl, 1994, for more details).

This paper qualifies this important claim by Wirl by introducing uncertainty. If the players do not know with certainty the politico-economic environment in the near and far future, and provided they are not too averse to risk, they might well depart from the overly cautious behaviour described in Wirl (1994). This is especially the case if they anticipate a favorable evolution of the environment. We apply our framework to the process of liberalization in oil exporting countries, and more specifically to Arab countries. The Arab Spring has shown the deep inequalities that characterize the Arab world. On one hand, there are ruling dynasties who usually control all types of economic and political activities. On the other, there is a majority of Arab citizens which are partially or totally excluded from relevant decision-making. A fundamental characteristic of these countries is the essential role played by the oil rents both on the political and economic grounds (see Caselli and Cunnigham, 2009). The larger these rents are, the bigger the incentives of the elites to stay in power and to block any initiative to open the political game ${ }^{3}$ In many Arab countries, starting with the Gulf emirates and kingdoms, a lot has been already done towards economic liberalization, notably in order to attract more foreign direct investment. However, no significant move has been made in favor of political liberalization (see

\footnotetext{
${ }^{3}$ Gylfason (2001) makes the point that the elites would eventually block human capital education to perpetuate themselves in power. As outlined by Boucekkine and Bouklia-Hassane (2011), this is certainly not the case of Tunisia, the starter in the Arab Spring uprising: more than $20 \%$ of the Tunisian budget has gone to public education in the last decade, much better than many advanced European countries.
} 
Dunne and Revkin, 2011, on Egypt) ${ }^{4}$ We shall consider a framework à la Wirl where political liberalization is the outcome of a piecewise deterministic differential game between a conservative elite and a reformist group: oil rents may be high or low (two states of nature). In the former state of nature, the elite is more reluctant to political liberalization. In this context, we revisit Wirl's findings and show how uncertainty alter the optimal strategies in the Markovperfect equilibria compared to the benchmark. Incidentally, we highlight some of the specific risks inherent in the current political libearlization process in Arab countries.

This paper is structured as follows. Section 2 introduces the dynamic model of political liberalization. Section 3 considers a setting with uncertainty and derives the MPE of a piecewise deterministic game. Finally, Section 4 concludes.

\section{Benchmark model}

In this section, the differential game on lobbying proposed by Wirl (1994) is adapted to the context of the Arab Spring. For the meantime, the case with no uncertainty is discussed. In the next section, we extend Wirl's model by considering a stochastic environment with two states of nature. Throughout the paper, we consider only symmetric games (in the precise sense of Wirl, see Section 2.1 just below). This is done for algebraic amenability, as no analytical solution is allowed outside this class of games.It goes without saying that players engaged in the political liberalization struggle in Arab countries do not have equal power since they do not have equal access to oil rents, etc 5 Nonetheless, the symmetric set-up adopted hereafter includes two important ingredients of the actual political liberalization game: the conservative elite is reluctant to political liberalization, while the reformist minority pushes for it. This reluctance is an increasing function of oil rents. The former point will be apparent in the stochastic extension of the benchmark described just below.

\footnotetext{
${ }^{4}$ Algeria is a case where even economic liberalization efforts have been tightly linked to the level of the oil barrel, as explained in Boucekkine and Bouklia-Hassane (2011).

${ }^{5}$ For example, in the Algerian case, the conservative elites benefit from the support of the powerful National Popular Army and the intelligence services (DRS).
} 


\subsection{The setup}

We consider two competing players (denoted as $i=1,2$ ) who engage in investment efforts $x_{1}$ and $x_{2}$. Player 1 is a reformist who exerts pressure towards greater political liberalization. On the other hand, Player 2 prefers a conservative system. In the context of the Arab Spring, Player 2 can be considered as the elite government who wants to retain the political status quo. Player 1 represents the groups who prefer regime change. The state of liberalization is measured by $z \epsilon(-\infty, \infty)$. As in Wirl, $z=0$ is the neutral level of political liberalization. Indeed, the following differential equation formally captures the evolution of $z$ in response to the efforts of players 1 and 2 :

$$
\dot{z}=x_{1}-x_{2},
$$

with $z(0)=z_{0}$ given. As a reformist, player 1 prefers a higher level of political liberalization. A high value of $z$, on the other hand, is not beneficial to the conservative stance of player 2 . Consequently, the investment $x_{1}$ of player 1 increases $z$, while player 2 exerts effort $x_{2}$ to lower $z$.

The benefit from the current level of liberalization is denoted by $\alpha_{i}(z)$ with: $\alpha_{1}(z)=a_{0}+a_{1} z+\frac{a_{2}}{2} z^{2}$ and $\alpha_{2}(z)=a_{0}-a_{1} z+\frac{a_{2}}{2} z^{2}$. We follow Wirl (1994) by qualifying this game as a symmetric one. The opposite signs of the second term in the players' benefit functions represent their antagonistic interests with regard to liberalization. Without loss of generality, we assume that $a_{1}>0$. We also assume that $a_{2} \leq 0$ to ensure concavity. Meanwhile, efforts $x_{1}$ and $x_{2}$ are also associated with cost $\gamma\left(x_{i}\right)=\frac{d}{2}\left(x_{i}\right)^{2}$.

Players maximize the present value of benefits from liberalization minus the associated costs, $F_{i}=\alpha_{i}(z)-\gamma\left(x_{i}\right)$. With an interest rate $r>0$, players choose effort levels to maximize the following objective function subject to the evolution of $z$ (Equation 1):

$$
\max _{x_{i}(t)} \int_{0}^{\infty} e^{-r t}\left\{\alpha_{i}(z(t))-\gamma\left(x_{i}(t)\right)\right\} d t
$$

Indeed, the solution to this differential game is essentially the same as the symmetric version found in Wirl (1994). Consequently, in the next subsection, we will merely summarize the resulting open-loop and feedback strategies. In Section 3 , we will provide a comprehensive solution to a game under uncertainty and provide analytical comparisons. 


\subsection{Open-loop and feedback strategies}

As mentioned above, this subsection provides an overview of the open-loop and Markov-perfect equilibrum (MPE) solutions to the political liberalization game with no uncertainty. Similar to Wirl (1994), the strategy pair $\left\{x_{1}^{O}(t), x_{2}^{O}(t), t \epsilon[0, \infty)\right\}$ comprises an open-loop Nash equilibrium (OLNE) if both strategies, which depend on $t$, maximize the respective objective functions of the players. In summary, the open-loop case (presented in the feedback form) at a symmetric equilibrium results to:

$$
\begin{aligned}
& x_{1}^{O}=\frac{a_{1}}{r d}+\frac{1}{4}\left[r-\sqrt{\left.\left(r^{2}-\frac{8}{d} a_{2}\right)\right] z}\right. \\
& \left.x_{2}^{O}=\frac{a_{1}}{r d}-\frac{1}{4}\left[r-\sqrt{(} r^{2}-\frac{8}{d} a_{2}\right)\right] z,
\end{aligned}
$$

which leads the system to a unique steady state characterized by:

$$
x_{1 \infty}^{O}=\frac{a_{1}}{r d}=x_{2 \infty}^{O} ; z_{\infty}=0 .
$$

While the open-loop equilibrium is time-consistent, it is not subgame perfect. That is, using open-loop strategies might not make sense when considering an anticipated change in the evolution of the game. Thus, following literature (Dockner, et al. 2000), feedback strategies are deemed suitable. Utilizing the usual Hamilton-Jacobi-Bellman (HJB) equations (refer to Wirl (1994), pg. 315 for a detailed discussion), the resulting MPE strategies in the case without regime switching are (the superscript $N$ is used here):

$$
\begin{aligned}
& x_{1}^{N}=\frac{6 a_{1}}{d\left[5 r+\sqrt{(} r^{2}-\frac{12}{d} a_{2}\right)}+\frac{1}{6}\left[r-\sqrt{\left.\left(r^{2}-\frac{12}{d} a_{2}\right)\right] z}\right. \\
& x_{2}^{N}=\frac{6 a_{1}}{d\left[5 r+\sqrt{\left(r^{2}-\frac{12}{d} a_{2}\right)}\right]}-\frac{1}{6}\left[r-\sqrt{\left.\left(r^{2}-\frac{12}{d} a_{2}\right)\right] z}\right.
\end{aligned}
$$

which leads the system to a steady state characterized by:

$$
x_{1 \infty}^{N}=\frac{6 a_{1}}{d\left[5 r+\sqrt{\left.\left(r^{2}-\frac{12}{d} a_{2}\right)\right]}\right.}=x_{2 \infty}^{N} ; z_{\infty}=0 .
$$

The strategies computed have some interesting implications. First, note that in the MPE, the strategy of player 1 is decreasing in $z$. This is in strong contrast to player 2. In terms of our political liberalization framework, it means that the reformist would exert less effort when the level of political freedom is rising. Meanwhile, the conservative will take the opposite way. Much more 
interestingly, one can use the previous feedback rules to conclude that $x_{i \infty}^{O}>$ $x_{i \infty}^{N}$, for $i=1,2$, which is the main result of Wirl's benchmark. That is, lobbying activities are lower in the MPE compared to the open loop, at least in the steady state. Therefore, the social cost of lobbying activities are less significant than one may expect. This finding is confirmed through some quantitative exercises ${ }^{6}$

\section{Political liberalization game under uncertainty}

We now consider the dynamic game of political liberalization under a setting with uncertainty.

\subsection{MPE of the piecewise deterministic game}

The symmetric case found in Wirl (1994) is extended by taking into account the possibility of regime switching. A stochastic differential game is analyzed. More specifically, we derive the Markov-perfect Nash equilibria of a piecewise deterministic game 7

The pay-offs of players 1 and 2 are altered to:

$$
\begin{aligned}
& F_{1}^{j}=a_{0}+a_{1}^{j} z+\frac{a_{2}}{2} z^{2}-\frac{d}{2}\left(x_{1}\right)^{2} \\
& F_{2}^{j}=a_{0}-a_{1}^{j} z+\frac{a_{2}}{2} z^{2}-\frac{d}{2}\left(x_{2}\right)^{2}
\end{aligned}
$$

Uncertainty is characterized in the coefficient representing the linear benefits incurred from liberalization, $a_{i}^{j}$. There exist two states of the world, denoted by $j$. In regime $1, a_{1}^{1}=\bar{a}_{1}$. On the other hand, $a_{1}^{2}=\underline{a}_{1}$ for regime 2 . We assume that $\underline{a}_{1}<\bar{a}_{1}$. In the context of the Arab Spring in predominantly oilrich economies, regime 1 can be the state when resource windfalls are high 8 Meanwhile, regime 2 can be considered as the scenario during which gains from oil are low. In regime 1, oil revenues are high, making player 2 even more reluctant to liberalization. This relatively higher reluctance translates into the fact that $\alpha_{2}(z)$ worsens in regime 1 , compared to regime 2 , due to a higher

\footnotetext{
${ }^{6}$ In the numerical cases studied by Wirl, the comparison is quantitatively striking. Indeed, the ratio of total lobbying expenditures in the MPE compared with the open loop is only around one third for the symmetric case, and even much less in some asymmetric configurations considered.

${ }^{7}$ We do not consider the piecewise open-loop equilibria as closed-form solutions for this case are rarely derived in literature (Dockner et al., 2000). For analytical tractability, we thus focus on feedback strategies.

${ }^{8}$ In most oil-dependent Arab countries, natural resource rents are usually received by the governing political elite (Caselli and Cunningham, 2009).
} 
$a_{1}$, in absolute terms. This means that, by symmetry, player 1's gains from liberalization are higher in the first regime. Furthermore, the probability to switch from regime 1 to 2 is denoted as $q_{12} \in(0,1)$. Similarly, the probability of switching from regime 2 to 1 is $q_{21} \in(0,1)$. Depending on the current regime and taking into account the switching probabilities, players maximize the discounted net payoffs in (7) subject to (1).

As discussed in Dockner et al. (2000), the HJB equations are modified and solved for each regime. The HJB equations for the piecewise deterministic game take the following form 9

$$
r V_{i}^{j}=\max _{x_{i}}\left\{F_{i}^{j}+\frac{\partial V_{i}^{j}}{\partial z} \dot{z}+q_{j,-j}\left[V_{i}^{-j}-V_{i}^{j}\right]\right\}
$$

Suppose we are in regime 1, the HJB equation for player 1 is denoted as:

$$
r V_{1}^{1}=\left\{\begin{array}{l}
\max _{x_{1}}\left\{a_{0}+a_{1}^{j} z+\frac{a_{2}}{2} z^{2}-\frac{d}{2}\left(x_{1}\right)^{2}+\left(B_{1}^{1}+C_{1}^{1} z\right)\left(x_{1}-x_{2}\right)+\right. \\
\left.+q_{12}\left[\left(A_{1}^{2}-A_{1}^{1}\right)+\left(B_{1}^{2}-B_{1}^{1}\right) z+\frac{\left(C_{1}^{2}-C_{1}^{1}\right)}{2} z^{2}\right]\right\}
\end{array}\right.
$$

where we guess that the value function has the following form

$$
V_{i}^{j}(z)=A_{i}^{j}+B_{i}^{j} z+\frac{C_{i}^{j}}{2} z^{2} i, j=1,2 .
$$

The first-order condition yields:

$$
x_{1}^{1}=\frac{B_{1}^{1}+C_{1}^{1} z}{d}
$$

Similarly, from player 2's HJB equation, we derive:

$$
x_{2}^{1}=-\frac{B_{2}^{1}+C_{2}^{1} z}{d}
$$

Substituting $x_{1}^{1}$ and $x_{2}^{1}$ by the expressions given in 10 and 11 in 9 , we obtain for player 1 (disregarding the constant terms):

$$
r\left(B_{1}^{1} z+\frac{C_{1}^{1}}{2} z^{2}\right)=\left\{\begin{array}{l}
a_{1}^{1} z+\frac{a_{2}}{2} z^{2}+\frac{1}{2 d}\left(B_{1}^{1}+C_{1}^{1} z\right)^{2}+ \\
+\frac{\left(B_{1}^{1}+C_{1}^{1} z\right)\left(B_{2}^{1}+C_{2}^{1} z\right)}{d}+q_{12}\left[\left(B_{1}^{2}-B_{1}^{1}\right) z+\frac{\left(C_{1}^{2}-C_{1}^{1}\right)}{2} z^{2}\right] .
\end{array}\right.
$$

\footnotetext{
${ }^{9}$ Compared to the general form of HJBs utilized in Wirl (1994), there is an additional (last) term which accounts for the possibility of uncertain regime switching from one regime, $j$, to the other, $-j$.
} 
Let's now proceed with the identification step. From the equation above, we have the following for player 1 :

$$
\begin{aligned}
& B_{1}^{1}\left(r+q_{12}-\frac{C_{1}^{1}+C_{2}^{1}}{d}\right)=a_{1}^{1}+\frac{B_{2}^{1} C_{1}^{1}}{d}+q_{12} B_{1}^{2} \\
& \frac{\left(C_{1}^{1}\right)^{2}}{2 d}-C_{1}^{1}\left(\frac{r+q_{12}}{2}-\frac{C_{2}^{1}}{d}\right)+q_{12} \frac{C_{1}^{2}}{2}+\frac{a_{2}}{2}=0
\end{aligned}
$$

Similarly, for player 2:

$$
\begin{gathered}
B_{2}^{1}\left(r+q_{12}-\frac{C_{1}^{1}+C_{2}^{1}}{d}\right)=-a_{1}^{1}+\frac{B_{1}^{1} C_{2}^{1}}{d}+q_{12} B_{2}^{2} \\
\frac{\left(C_{2}^{1}\right)^{2}}{2 d}-C_{2}^{1}\left(\frac{r+q_{12}}{2}-\frac{C_{1}^{1}}{d}\right)+q_{12} \frac{C_{2}^{2}}{2}+\frac{a_{2}}{2}=0
\end{gathered}
$$

Suppose instead players are in regime 2. Following the same methodology as before, we get:

$$
\begin{aligned}
& B_{1}^{2}\left(r+q_{21}-\frac{C_{1}^{2}+C_{2}^{2}}{d}\right)=a_{1}^{2}+\frac{B_{2}^{2} C_{1}^{2}}{d}+q_{21} B_{1}^{1} \\
& \frac{\left(C_{1}^{2}\right)^{2}}{2 d}-C_{1}^{2}\left(\frac{r+q_{21}}{2}-\frac{C_{2}^{2}}{d}\right)+q_{21} \frac{C_{1}^{1}}{2}+\frac{a_{2}}{2}=0 \\
& B_{2}^{2}\left(r+q_{21}-\frac{C_{1}^{2}+C_{2}^{2}}{d}\right)=-a_{1}^{2}+\frac{B_{1}^{2} C_{2}^{2}}{d}+q_{21} B_{2}^{1} \\
& \frac{\left(C_{2}^{2}\right)^{2}}{2 d}-C_{2}^{2}\left(\frac{r+q_{21}}{2}-\frac{C_{1}^{2}}{d}\right)+q_{21} \frac{C_{2}^{1}}{2}+\frac{a_{2}}{2}=0
\end{aligned}
$$

To identify the parameters relevant for each player, we first consider the system 14, 16, , 18) and (20). Let us assume that $C_{i}^{j}$ parameters are identical for players in any regime $j: C_{1}^{j}=C_{2}^{j}$ for $j=1,2$. Substituting these relationships into our system, we are left with a system of two equations

$$
\begin{aligned}
& \frac{3}{d}\left(C_{1}^{1}\right)^{2}-\left(r+q_{12}\right) C_{1}^{1}+q_{12} C_{1}^{2}+a_{2}=0 \\
& \frac{3}{d}\left(C_{1}^{2}\right)^{2}-\left(r+q_{21}\right) C_{1}^{2}+q_{21} C_{1}^{1}+a_{2}=0
\end{aligned}
$$

in two unknowns $\left(C_{1}^{1}, C_{1}^{2}\right)$. Taking the difference between these two equations, one obtains:

$$
\frac{3}{d}\left[\left(C_{1}^{1}\right)^{2}-\left(C_{1}^{2}\right)^{2}\right]-\left(r+q_{12}+q_{21}\right)\left(C_{1}^{1}-C_{1}^{2}\right)=0
$$

Observing (22), two cases are possible: $1 . C_{1}^{1} \neq C_{1}^{2}$ and 2. $C_{1}^{1}=C_{1}^{2}$. 
- First, suppose that $C_{1}^{1} \neq C_{1}^{2}$. Then, 22 simplifies to:

$$
C_{1}^{2}=\frac{d}{3}\left(r+q_{12}+q_{21}\right)-C_{1}^{1}
$$

Using (23), the first equation in (21), can be rewritten as

$$
\frac{3}{d}\left(C_{1}^{1}\right)^{2}-\left(r+2 q_{12}\right) C_{1}^{1}+\frac{d q_{12}}{3}\left(r+q_{12}+q_{21}\right)+a_{2}=0
$$

Assuming that $\Delta_{1}=r^{2}-\frac{12}{d} a_{2}-4 q_{12} q_{21}>0$, two solutions thus exist

$$
\left.\begin{array}{l}
C_{1}^{1-}=C_{2}^{1-}=C^{1-}=\frac{d}{6}\left(r+2 q_{12}-\sqrt{\left(\Delta_{1}\right)}\right) \\
C_{1}^{1+}=C_{2}^{1+}=C^{1+}=\frac{d}{6}\left(r+2 q_{12}+\sqrt{(} \Delta_{1}\right)
\end{array}\right)
$$

each corresponding to a particular $C_{1}^{2}$

$$
\begin{aligned}
& C_{1}^{2+}=C_{2}^{2+}=C^{2+}=\frac{d}{6}\left(r+2 q_{21}+\sqrt{\left(\Delta_{1}\right)}\right) \\
& \left.C_{1}^{2-}=C_{2}^{2-}=C^{2-}=\frac{d}{6}\left(r+2 q_{21}-\sqrt{(} \Delta_{1}\right)\right)
\end{aligned}
$$

Specifically, solutions are $\left(C^{1-}, C^{2+}\right)$ and $\left(C^{1+}, C^{2-}\right)$.

- Second, consider that $C_{1}^{1}=C_{1}^{2}$. Then, the $C$ parameter is the same for both regimes and for both players. It is equal to

$$
\begin{aligned}
& C^{+}=\frac{d}{6}\left(r+\sqrt{\left(\Delta_{2}\right)}\right) \\
& C^{-}=\frac{d}{6}\left(r-\sqrt{\left(\Delta_{2}\right)}\right)
\end{aligned}
$$

with $\Delta_{2}=r^{2}-\frac{12}{d} a_{2}>0$ if $\Delta_{1}>0$. In this case, players' response to a change in $z$ is similar to one of Wirl, obtained in the problem with no uncertainty (Section $2)$.

We now turn to the identification of $B$-parameters by solving the system 13), (15), 17), 19). Guessing that $B_{2}^{i}=-B_{1}^{i}$ for $i=1,2$, this system simplifies to:

$$
\begin{aligned}
& B_{1}^{1}\left(r+q_{12}-\frac{C^{1}}{d}\right)=a_{1}^{1}+q_{12} B_{1}^{2} \\
& B_{1}^{2}\left(r+q_{21}-\frac{C^{2}}{d}\right)=a_{1}^{2}+q_{21} B_{1}^{1}
\end{aligned}
$$

Combining these equations, we obtain the general solution, for $B$ coefficients:

$$
B_{1}^{1}=\frac{\left(r+q_{21}-\frac{C^{2}}{d}\right) a_{1}^{1}+q_{12} a_{1}^{2}}{\left(r-\frac{C^{2}}{d}\right)\left(r-\frac{C^{1}}{d}\right)+q_{12}\left(r-\frac{C^{2}}{d}\right)+q_{21}\left(r-\frac{C^{1}}{d}\right)}
$$




$$
B_{1}^{2}=\frac{\left(r+q_{12}-\frac{C^{1}}{d}\right) a_{1}^{2}+q_{21} a_{1}^{1}}{\left(r-\frac{C^{2}}{d}\right)\left(r-\frac{C^{1}}{d}\right)+q_{12}\left(r-\frac{C^{2}}{d}\right)+q_{21}\left(r-\frac{C^{1}}{d}\right)}
$$

In summary, depending on the particular $C$ considered, there are four potential solutions to our uncertain problem. The first type of solution exhibits identical $C$-parameters in both regimes. So, each player adapts her strategy to changes in the liberalization level in the same way, whatever the regime. In this sense, this solution looks like Wirl's outcome. There also exist solutions for which $C$ s parameters change from one regime to the other, which gives rise to more considerable differences in players' behavior. The next section investigates the properties of these two types of solutions. Particular attention will be paid to the impact of uncertainty on players' strategies through the comparison between solutions for the cases with and without uncertainty.

\subsection{Markov Perfect Equilibria with regime-independent responses to political liberalization}

Wirl (1994) has a unique MPE in his deterministic problem. Indeed, he uses a stability argument to select, among the two possible values of $C$ given in (27), the negative one. For the sake of comparison, we report players' strategies at our MPE with identical $C \mathrm{~s}$, given that $C=C^{-}\left(\right.$and $\left.\Delta_{2}=r^{2}-\frac{12}{d} a_{2}\right){ }_{10}^{10}$

Proposition 1 Players' efforts, at MPE, are

$$
\begin{aligned}
& x_{1}^{1}=\frac{6\left[\left(5 r+6 q_{21}+\sqrt{\Delta_{2}}\right) a_{1}^{1}+6 q_{12} a_{1}^{2}\right]}{d\left[5 r+\sqrt{\Delta_{2}}\right)\left[\left[5 r+\sqrt{\Delta_{2}}+6\left(q_{12}+q_{21}\right)\right]\right.}+\frac{1}{6}\left[r-\sqrt{\Delta_{2}}\right] z, \\
& \left.x_{2}^{1}=\frac{6\left[\left(5 r+6 q_{21}+\sqrt{\Delta_{2}}\right) a_{1}^{1}+6 q_{12} a_{1}^{2}\right]}{d\left[5 r+\sqrt{\Delta_{2}}\right]\left[5 r+\sqrt{\Delta_{2}}+6\left(q_{12}+q_{21}\right)\right.}\right]-\frac{1}{6}\left[r-\sqrt{\Delta_{2}}\right] z, \\
& \left.x_{1}^{2}=\frac{6\left[\left(5 r+6 q_{12}+\sqrt{\Delta_{2}}\right) a_{1}^{2}+6 q_{21} a_{1}^{1}\right]}{d\left[5 r+\sqrt{\Delta_{2}}\right]\left[5 r+\sqrt{\Delta_{2}}+6\left(q_{12}+q_{21}\right)\right.}\right]+\frac{1}{6}\left[r-\sqrt{\Delta_{2}}\right] z, \\
& \left.x_{2}^{2}=\frac{6\left[\left(5 r+6 q_{12}+\sqrt{\Delta_{2}}\right) a_{1}^{2}+6 q_{21} a_{1}^{1}\right]}{d\left[5 r+\sqrt{\Delta_{2}}\right]\left[5 r+\sqrt{\Delta_{2}}+6\left(q_{12}+q_{21}\right)\right.}\right]-\frac{1}{6}\left[r-\sqrt{\Delta_{2}}\right] z .
\end{aligned}
$$

For each regime separately, the dynamics drive the system toward a steady state with:

$$
\begin{gathered}
z_{\infty}^{1}=z_{\infty}^{2}=0, x_{i \infty}^{1}=\frac{6\left[\left(5 r+6 q_{21}+\sqrt{\Delta_{2}}\right) a_{1}^{1}+6 q_{12} a_{1}^{2}\right]}{\left.d\left[5 r+\sqrt{\Delta_{2}}\right)\right]\left[5 r+\sqrt{\Delta_{2}}+6\left(q_{12}+q_{21}\right)\right]} \\
\text { and } x_{i \infty}^{2}=\frac{6\left[\left(5 r+6 q_{12}+\sqrt{\Delta_{2}}\right) a_{1}^{2}+6 q_{21} a_{1}^{1}\right]}{d\left[5 r+\sqrt{\Delta_{2}}\right]\left[5 r+\sqrt{\Delta_{2}}+6\left(q_{12}+q_{21}\right)\right]} \text { for } i=1,2 .
\end{gathered}
$$

\footnotetext{
${ }^{10}$ In our stochastic framework, we also have a solution corresponding to $C=C^{+}$, which can't be eliminated using the stability argument. However, straightforward calculations reveal that this solution has undesirable features: the level of liberalization goes to infinity, which implies that the liberalization effort of player 2 goes to $-\infty$ (in the absence of nonnegativity constraint on $x$ ). That's why we choose to focus on the other solution, that is also more consistent with Wirl's outcome.
} 
One can check that strategies in 30 reduce to Wirl-type MPE, $\left(x_{1}^{N}, x_{2}^{N}\right)$ defined in (5), when assuming $q_{12}=q_{21}=0, a_{1}^{2}=a_{1}^{1}=a_{1}$. Besides, at first glance, these strategies share similarities with the ones of the deterministic situation. In particular, for the solution with identical $C$ s, the effort of player 1 is always decreasing in $z$. Regardless of the regime, the opposite holds for player 2. When the level of liberalization is higher, player 1 would have less incentive to call for reforms as the system is already more favorable to his interests. On the other hand, a higher $z$ hurts the conservative stance of player 2. Hence, in order to counteract this, he exerts more effort.

There are however differences between equilibrium strategies found above and those derived for the Wirl-type, symmetric case in Section 2.2. Indeed, the existence of uncertainty plays an integral role in determining the effort levels of players. In what follows, results found in 2.2 and 3.1 are compared analytically. For ease of notation, we again denote "MPE" as the ones found for the uncertain case (with identical and different $C$ s) and "Wirl-type MPE" for the certain case. With $a_{1}^{1}>a_{1}^{2}$, the following proposition can be established.

Proposition 2 - MPE with identical Cs vs. Wirl-type MPE: $x_{i}^{N}>$ $x_{i}^{j}$ for $i=1,2$ and $j=1,2$ iff the deterministic economy is associated with $a_{1}=a_{1}^{1}$. The opposite holds, that is $x_{i}^{N}<x_{i}^{j}$ iff $a_{1}=a_{1}^{2}$.

- MPE with identical Cs vs. OLNE at the steady state: When $a_{1}=a_{1}^{1}$, it is straightforward that $x_{i \infty}^{O}>x_{i \infty}^{j}$ for all $i$ and all $j$ since $x_{i \infty}^{O}>x_{i \infty}^{N}$ and $x_{i}^{N}>x_{i}^{j}$ for all $z$. When $a_{1}=a_{1}^{2}, x_{i \infty}^{j}>x_{i \infty}^{O}$, for all $i$, for all $j$, if $a_{1}^{2}<\hat{a}_{1}^{2}$ with

$$
\hat{a}_{1}^{2}=a_{1}^{1} \times \frac{36 r q_{21}}{\left(5 r+{\sqrt{\Delta_{2}}}_{2}\right)\left(5 r+{\left.\sqrt{\Delta_{2}}+6\left(q_{12}+q_{21}\right)-36 r q_{12}\right)}\right.}
$$

The proof is relegated to the appendix (see Appendix A). Proposition 2 has several implications. First, recall that from (30) it can be shown that $x_{i}^{1}>x_{i}^{2}$. In the MPE with identical Cs, the efforts of players are greater when they are in a state with high windfalls than when they are in the low regime.This finding is analogous to taking $\frac{\partial x_{i}^{N}}{\partial a_{1}}$ for the deterministic, Wirl-type case. An incremental increase in the coefficient representing the linear benefits from the liberalization level $z$ implies an increase in the effort levels. All other things constant, the reformist's investment will rise when $a_{1}$ goes up. Knowing that this increase 
in $a_{1}$ may hurt him, the conservative will invest more to counteract player 1's action.

Second, the impact of uncertainty on the comparative relationship between the MPE with identical $C$ s and the Wirl-type MPE is not clear-cut. Uncertainty lowers the equilibrium investment levels in comparison to the case when $a_{1}$ is surely in a high state. Assume that players are in Regime 1 at the present. Knowing that there is a probability that the regime will shift to a setting with low windfalls, players have less incentive to impact liberalization (i.e. relative to the scenario when they are certain that they will always be in Regime 1). Consequently, we find the following relationship: $x_{i}^{N}>x_{i}^{j}$ when $a_{1}=a_{1}^{1}$. Contrast this to the case when $a_{1}=a_{1}^{2}$. Indeed, the opposite occurs when comparing our MPE to the Wirl-type MPE for the low state. Suppose players are in Regime 2. Since there is a possibility that the regime will alter to a system with higher windfalls, they invest more. Indeed, because of an anticipation of a potential shift to the high state, the MPE with identical Cs is higher relative to the Wirl-type MPE for the low state: $x_{i}^{N}<x_{i}^{j}$.

Third, the steady state levels of the MPE with identical $C$ s and the OLNE can be compared. When $a_{1}=a_{1}^{1}$ (high state regime), the open-loop equilibrium investments are greater than the MPE with identical Cs when $z_{\infty}=0$. Similar to the deterministic case, players exert relatively less effort into affecting the level of political liberalization. This is because feedback strategies among players are characterized by a dynamic retaliation mechanism. For instance, whenever Player 1 triumphs in shifting the liberalization level towards his favor, she knows that Player 2 will retaliate more. As Wirl (1994) argued, this common knowledge actually deters aggressive strategies. However, this is not the case when $a_{1}=a_{1}^{2}$. In particular, the above-mentioned observation does not apply when $a_{1}^{2}$ is low enough. At the steady state, the OLNE for the symmetric case in the low state is below the MPE with identical $C$ s. Even in the potential presence of retaliation, the existence of uncertainty induces players to exert more effort compared to the OLNE in the low state. Remember that for the Wirl-type solution, players know that they will always be in the low state. Compare this when they are facing uncertainty. That is, suppose they are currently in regime 2. The possibility of shifting to regime 1 may imply more aggressive investment. As a result, the cost of lobbying along the MPE equilibria under uncertainty 
might be significantly increased with respect to Wirl's deterministic benchmark.

In the context of the political libearlization process at stake in Arab countries, this highlights the property that the uncertainty related to the oil rents, a fundamental ingredient of the game in these countries, is likely to generate significant social costs inherent in the game contrary to what is predicted by the standard deterministic theory. The larger this uncertainty, the larger the social costs as strategies will become more aggressive. In other words, independently of the well-known economic costs associated with volatility of resource prices in exporting countries, this volatility will make the political liberalization process itself more costly adding to the latter. Another complication of uncertainty is the emergence of alternative strategies which do not show up in deterministic frameworks.

\subsection{Markov perfect equilibria with regime-driven responses to changes in liberalization}

The solution discussed in the preceding section can be contrasted with a $\left(C^{1}, C^{2}\right)$ type of solution, with $C^{1}, C^{2}$ given in $\left.25-26\right)$. In this case, players' reaction to a change in the liberalization level is dependent on the particular regime in which the economy is lying. Let us see how this regime-driven reaction impacts on the properties of the solution. For that purpose, in the next proposition we present equilibrium strategies for the case where $C^{1}=C^{1-}, C^{2}=C^{2+}$, and $\Delta_{1}=r^{2}-\frac{12}{d} a_{2}-4 q_{12} q_{21}$. Then, a discussion about the features of this solution and how it compares to the Wirl-type MPE is conducted 11

Proposition 3 Suppose there exists a MPE with regime-driven response to changes in liberalization, then the strategies are given by

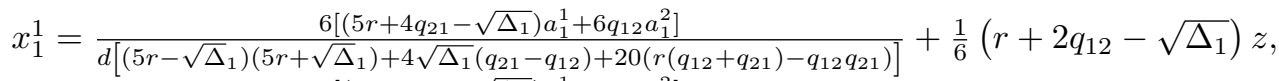

$$
\begin{aligned}
& x_{2}^{1}=\frac{6\left[\left(5 r+4 q_{21}-\sqrt{\Delta_{1}}\right) a_{1}^{1}+6 q_{12} a_{1}^{2}\right]}{d\left[\left(5 r-\sqrt{\Delta_{1}}\right)\left(5 r+\sqrt{\left.\Delta_{1}\right)+4 \sqrt{\Delta_{1}}\left(q_{21}-q_{12}\right)+20\left(r\left(q_{12}+q_{21}\right)-q_{12} q_{21}\right)}\right]\right.}-\frac{1}{6}\left(r+2 q_{12}-\sqrt{\Delta_{1}}\right) z, \\
& x_{1}^{2}=\frac{6\left[\left(5 r+4 q_{12}+\sqrt{\Delta_{1}}\right) a_{1}^{2}+6 q_{21} a_{1}^{1}\right]}{d\left[\left(5 r-{\left.\sqrt{\Delta_{1}}\right)\left(5 r+\sqrt{\Delta}_{1}\right)+4 \sqrt{\Delta_{1}}\left(q_{21}-q_{12}\right)+20\left(r\left(q_{12}+q_{21}\right)-q_{12} q_{21}\right)}\right)\right.}+\frac{1}{6}\left(r+2 q_{21}+\sqrt{\Delta_{1}}\right) z,
\end{aligned}
$$

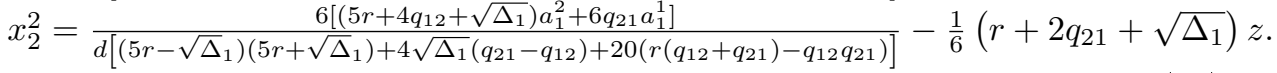

\footnotetext{
${ }^{11}$ The conclusions drawn from analysis of the other MPE candidate, corresponding to $\left(C^{1+}, C^{2-}\right)$, are qualitatively similar to the ones obtained for $\left(C^{1-}, C^{2+}\right)$. For this reason, this case is not dealt with by the subsequent study.
} 
It's out of the scope of this paper to provide a full analysis of the ergodicity properties of the solutions. However, we can mention some distinctive features of the alternative MPEs through a separate analysis of our two regimes involved. Paying attention to regime 2, from (33), we observe that the limit value of $z$ is infinite, positive or negative depending on the sign of the initial level of liberalization $z_{0}$. As mentioned in footnote 11, this means that if the economy were to stay in regime 2 for a sufficiently long interval of time, then player 2's effort would become negative. It is also worth checking how the system behaves in regime 1. Indeed, it turns out that regime 1's dynamics are qualitatively similar to the ones of regime 2 when one assumes

$$
-3 \frac{a_{2}}{d}<q_{12}\left(r+q_{12}+q_{21}\right)
$$

because under this condition, $C^{1-}, C^{2+}>0$.

Given that the economy randomly switches from regime 1 to regime 2, and vice-versa, one may prefer imposing the opposite of (34). In that case, dynamics of regime 1 are similar to the ones holding at the Wirl-type MPE or at our MPE with identical response to changes in liberalization. It implies that the limit value of $z$ would be zero whereas $x_{1}^{1}$ and $x_{2}^{1}$ would reach finite values ${ }^{12}$

Several observations can be noted from the comparison of the solution in Proposition 3 and the Wirl-type MPE.

First, the impact of an increase in $z$ on effort levels is very different from what observed for the Wirl-type MPE (and the MPE with identical $C$ s). From (33), notice the obvious effect of a higher $z$ on the efforts of players in the second regime. In regime 2, player 1's (player2) investment increases (decreases) with $z$. Regardless of the switching probabilities, regime 2 always induces the abovementioned results. The low state of $a_{1}^{2}$ gives greater incentive to player 1 to exert more effort when $z$ increases. This is because he wants to take more advantage from political liberalization. There exists a form of intensified reinforcement. In contrast, when $z$ goes up, player 2 knows it becomes more favorable to player 1. Knowing that exerting effort is costly, it is actually strategic for player 2 to lessen his investment. When $z$ already acquired a much higher level, it might be more difficult for him to shift the system to his favor. There is deterrence in his incentive to change the system.

\footnotetext{
${ }^{12}$ Thus, in some sense, the dynamical system valid in regime 1 offsets the explosive trend of regime 2 .
} 
From the discussion above, the reasoning is less obvious for regime 1 . The findings are similar to those in regime 2 only when Condition (34) is satisfied. This is all the more likely, given that switching probabilities are high enough. In this case, the strategy of player 1 increases with respect to the state $z$ while the opposite is relevant for player 2. Indeed, when the $C$-parameters are different, the impact of uncertainty becomes more prominent. Interestingly, the results become the inverse of those observed for the deterministic, Wirl type MPE. Suppose players are currently in regime 1 . Given a relatively high probability of switching to regime 2, an incremental increase in $z$ induces player 1 to exert more effort. This happens because player 1 knows that she obtains less linear benefits from liberalization in regime 2 (due to lower $a_{1}$ ). With the anticipation that he might be in regime 2 the next period, player 1 tries to compensate and invests more aggressively in the favorable regime 1 . In contrast, player 2's effort in regime 1 decreases with $z$ when the likelihood of switching to regime 2 is high enough. Given that regime 2 is more favorable to player 2 , i.e. $a_{1}$ is reasonably lower, then he has less incentive to invest in regime 1.

If Condition (34) does not hold, then the results in regime 1 are similar to those found in the MPE with identical $C$ s and the Wirl-type MPE. Indeed, when players are in regime 1 and the probability of switching to regime 2 is rather low, their incentives are different from those observed when they are regime 2. Knowing that there is a higher likelihood that he will stay in the favorable regime 1, player 1 invests less when political liberalization is more prevalent. Meanwhile, a higher $z$ combined with being in regime 1 harms the other player more. Player 2 mitigates this by trying to shift the system to his favor, i.e. exert more effort against liberalization.

Finally, it is worth noting that when $a_{2}=0$, the MPE strategies are constant for the Wirl-type MPE and the solution with identical $C$ s. However, because switching probabilities appear in the solution for different $\mathrm{Cs}$, this is not the case for the MPE with dissimilar $C$-parameters. The strategies of players in the MPE with different $C$ s still vary with $z$. Taking into account the role of uncertainty (i.e. $C$ varies for each regime), the effort levels do not remain constant. Player 1 's (player 2) effort is always increasing (decreasing) in $z$. The explanation for this result utilizes a similar logic as above. 


\section{Conclusion}

In this paper, we have developed a dynamic game of political liberalization under uncertainty. This is done by using the context of the Arab Spring in resource-rich countries. It has been observed that effort levels of reformists (those who benefit from greater liberalization) and conservatives (those who are against liberalization) tend to differ depending on the setup of the game. In the case with no uncertainty, the strategy of the reformist decreases with respect to the liberalization level while the opposite is true for the conservative. In striking difference, opposite results were observed in the case with uncertainty. When the regime switching probabilities are high enough, the reformist's effort increases with respect to the state $z$. On the other hand, the conservative's investment decreases with intensified political liberalization. In the presence of uncertainty and greater likelihood of regime shift, an increase in $z$ reinforces the reformist's incentive to induce change. In contrast, when $z$ goes up, the conservative is in a less favorable position and is surprisingly deterred from altering the system. Finally, it is also shown that in certain circumstances, the cost of lobbying might be significantly increased under uncertainty with respect to Wirl's benchmark. In the context of the political libearlization process at stake in Arab countries, this means that the uncertainty related to the oil rents is likely to generate significant social costs. As mentioned before, this implies that increased rents volatility will make the political liberalization process itself more costly adding to the well-known economic costs associated with volatility of resource prices in exporting countries.

Subject to analytical tractability, the present model may be extended in the following directions. First, one may introduce uncertainty in the cost functions,

e.g. it is less costly to invest in regime 1 than in 2 . Second, one may explore a different stochastic environment by incorporating a Wiener-type process that may affect the evolution of political liberalization. 


\section{References}

[1] Becker G (1983) A theory of competition among pressure groups for political influence. Quarterly Journal of Economics 98: 371-400

[2] Boucekkine R, Bouklia-Hassane R (2011) Rente, corruption et violence: l'ïœmergence d'un ordre nouveau dans les pays arabes ?. Regards Economiques 92. Available at http://sites.uclouvain.be/econ/ Regards/Archives/RE092.pdf.

[3] Caselli F, Cunningham T (2009) Leader behaviour and the natural resource curse. Oxford Economic Papers 61: 628-650

[4] Dockner E, Jorgensen S, Long N, Sorger G (2000) Differential games in economics and management science. Cambridge University Press, Cambridge.

[5] Dunne M, Revkin M (2011) Egypt: How a lack of political reform undermined economic reform. Carnegie Endowment for International Peace. Available at http://carnegie-mec.org/publications/?fa=42710.

[6] Gylfason T (2001) Natural resources, education, and economic development. European Economic Review 45: 847-859

[7] Kohli I, Singh N (1999) Rent-seeking and rent-seeking with asymmetric effectiveness of lobbying. Public Choice 99:275-98

[8] Linster B (1994) Cooperative rent-seeking. Public Choice 81: 23-34

[9] Mehlum H, Moene K, Torvik R (2006) Cursed by resources or institutions? The World Economy 29: 1117-1131

[10] Ross ML (2001) Timber booms and institutional breakdown in Southeast Asia. Cambridge University Press, New York.

[11] Tulock G (1967) The welfare costs of tariffs, monopolies, and theft. Western Economic Journal 5:224-232

[12] Wirl F (1994) The dynamics of lobbying: a differential game. Public Choice 80: $307-323$ 


\section{Appendix}

\section{A Proof of Proposition 1}

\section{A.1 MPE with identical $C$ s vs. Wirl-type MPE}

Here we compare the MPE in the deterministic case is (Wirl-type results):

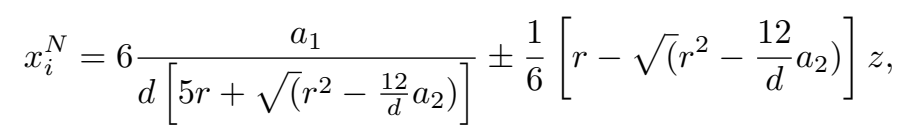

with the MPE obtained with uncertain regime switching and identical $C \mathrm{~s}$. In regime 1 ,

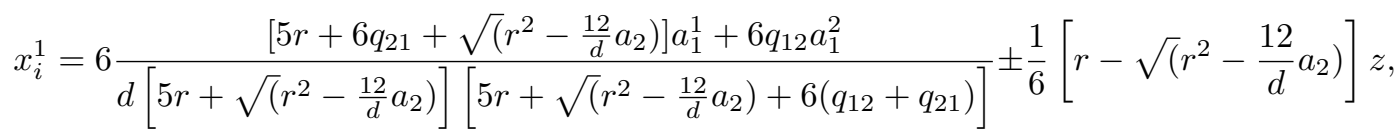

and in regime 2 ,

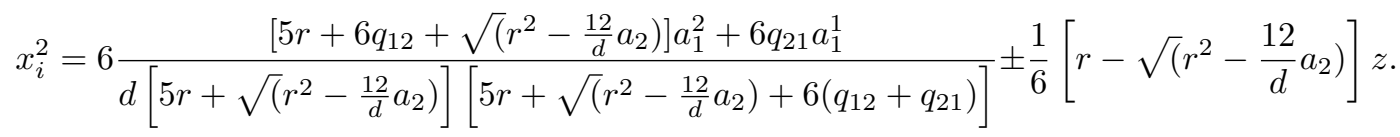

- Let us first consider that the deterministic $a_{1}$ is the high one: $a_{1}=a_{1}^{1}$. Then, it is trivial to show that $x_{i}^{j}<x_{j}^{N} \Leftrightarrow a_{1}^{2}<a_{1}^{1}$ for $i, j=1,2$ and for all $z$. This is satisfied by definition.

- Next, suppose that the deterministic $a_{1}$ is the one corresponding to regime 2: $a_{1}=a_{1}^{2}$. Then, one can check easily that $x_{i}^{j}>x_{i}^{N} \Leftrightarrow a_{1}^{2}<a_{1}^{1}$ for $i, j=1,2$ and for all $z$, which is true by definition.

\section{A.2 MPE with identical $C$ s vs. OLNE at the steady state}

Again, we make a distinction between two cases, depending on whether the deterministic $a_{1}$ is the high one or not. Following Wirl (1994), attention is paid only to the steady state.

When $a_{1}=a_{1}^{1}$, the comparison is straightforward: from what we learnt in the preceding appendix, we know that $x_{i}^{j}<x_{i}^{N}$ for all $z$. In particular, it holds that $x_{i \infty}^{j}<x_{i \infty}^{N}$ (recall that in both cases, $z_{\infty}=0$ ). In addition, Wirl (1994) has shown that $x_{i \infty}^{N}<x_{i \infty}^{O}$. So, we have $x_{i \infty}^{j}<x_{i \infty}^{O}$ for all $i, j=1,2$. 
When $a_{1}=a_{1}^{2}$, the comparison is less obvious because, at the same time, $x_{i \infty}^{j}>x_{i \infty}^{N}$ and $x_{i \infty}^{N}<x_{i \infty}^{O}$. In regime 1 , from the definition of the open-loop solution (see 3), $x_{i \infty}^{1}<x_{i \infty}^{O} \Leftrightarrow$

$$
\left[\left(5 r+{\sqrt{\Delta_{2}}}\right)\left(5 r+6\left(q_{12} q_{21}\right)+{\sqrt{\Delta_{2}}}\right)-36 r q_{12}\right] a_{1}^{2}<6 r\left(5+6 q_{21}+\sqrt{\Delta_{2}}\right) a_{1}^{1} .
$$

Note that the coefficient in the LHS is larger than the one in the RHS. So, given that $a_{1}^{1}>a_{1}^{2}, x_{i \infty}^{1}<x_{i \infty}^{O}$ is equivalent to

$$
a_{1}^{2}<\frac{6 r\left(5+6 q_{21}+{\sqrt{\Delta_{2}}}\right)}{\left[\left(5 r+{\sqrt{\Delta_{2}}}_{2}\right)\left(5 r+6\left(q_{12} q_{21}\right)+{\sqrt{\Delta_{2}}}\right)-36 r q_{12}\right]} a_{1}^{1},
$$

this defines an upper bound $\tilde{a}_{1}^{2}$ on the coefficient $a_{1}$ valid in the low regime.

In regime 2, following the same approach, we obtain that $x_{i \infty}^{2}<x_{i \infty}^{O} \Leftrightarrow$

$$
\left[\left(5 r+{\sqrt{\Delta_{2}}}_{2}\right)\left(5 r+6\left(q_{12} q_{21}\right)+{\sqrt{\Delta_{2}}}_{2}\right)-6 r\left(5 r+6 q_{12}+{\sqrt{\Delta_{2}}}_{2}\right)\right] a_{1}^{2}<36 r q_{21} a_{1}^{1},
$$

the coefficient in the LHS being again larger than the one in the RHS. Hence, $x_{i \infty}^{2}<x_{i \infty}^{O}$ is equivalent to

$$
a_{1}^{2}<\frac{36 r q_{21}}{\left[\left(5 r+{\sqrt{\Delta_{2}}}\right)\left(5 r+6\left(q_{12} q_{21}\right)+{\sqrt{\Delta_{2}}}\right)-36 r q_{12}\right]} a_{1}^{1},
$$

this defines a second boundary $\hat{a}_{1}^{2}$ on the coefficient $a_{1}$ valid in the low regime.

Now, given that $\tilde{a}_{1}^{2}>\hat{a}_{1}^{2}, a_{1}^{2}<\hat{a}_{1}^{2}$ implies that $x_{i \infty}^{j}<x_{i \infty}^{O}$ for $i, j=1,2$ when $a_{1}=a_{1}^{2}$.

This completes the proof. 